\title{
Photonic Band Gap in One-Dimensional Ternary Metal-Dielectric Photonic Crystal
}

\author{
*G. N. Pandey \\ Department of Physics, Amity Institute of Applied Sciences, Amity University, Noida (U.P.), India \\ Corresponding Author: G. N. Pandey
}

\begin{abstract}
In this present communication, we have theoretically studied the photonic band gap in ternary metal-dielectric photonic crystal which can be significantly enlarged when air is considered as a dielectric constant. All the theoretical analyses are based on the transfer matrix method together with the Drude model of metal. In this paper we have consider the effect of the variation of the thickness of the metal on the reflection bands of ternary metallic-dielectric photonic crystal (MDPC). We also observed that the enlargement of band gap in MDPC is due to the addition of increase of the thickness of metallic film at normal incidence.
\end{abstract}

\section{INTRODUCTION}

Nowadays the periodic arrangement of layers having different materials is called a photonic crystal. If this periodic arrangement is in the order of the wavelength of light, then for some range of wavelength of the light cannot pass through the crystal. This range of wavelength is called the photonic band gap (PBG) or forbidden band gap for the electromagnetic wave [1-5]. The photonic crystals are also known as the electromagnetic wave band gap materials because the electromagnetic wave cannot propagate through such photonic crystal if the incident wavelength is equivalent to the thickness of the unit cell of the crystals. Photonic crystals are a class of artificial periodic composite materials which exhibit electromagnetic band gap (i.e. frequency regime where the EM wave cannot propagate), this band gap property makes the PCs very important for the manipulation of EM wave; they can act for the EM wave in the same way as the semiconductors for the propagation of electrons. The photonic crystals are the artificial structures which are made up by combining the large numbers of the dielectric/metals materials. The metal has important characteristic in optics, if the thickness of metal is small than the skin depth, the EMW can transmit and if the thickness of metal large than skin depth, the EMW cannot transmit. Such metallic has studied for the telecommunication by studying the transmittance and reflectance of the periodic media. The optical properties of the metal is considered from Drude model of metal, the reflectance and transmission is calculated by using simple transfer matrix method [68]. G.N. Pandey et al. have been theoretically studied omni-directional reflection bands in one dimensional plasma photonic crystal in TE- and TM-modes and the optical properties of plasma photonic crystal. G N Pandey et al. have also studied the reflectance and transmittance behaviors of superconductor dielectric photonic crystal at different incidence angle and at different temperatures [9-16]. In this paper the photonic band structure in the visible and near infrared for a ternary metal dielectric photonic crystal has been theoretically investigated. It has been observed that the ternary metal dielectric photonic crystal has a superior feature in the enhancement of the high reflectance range with the Ag metal. In the normal incidence, the high reflectance range can be significantly enlarged for the thicker metal.

\section{Theory and Physical model}

A schematic diagram of a one-dimensional ternary metal dielectric photonic crystal (MDPC) of $\mathrm{N}$ - periodic multilayer may be considered when in each period of the metallic layer 2 is sandwiched by two dielectric layers 1 and 3 which are shown in fig.1. 


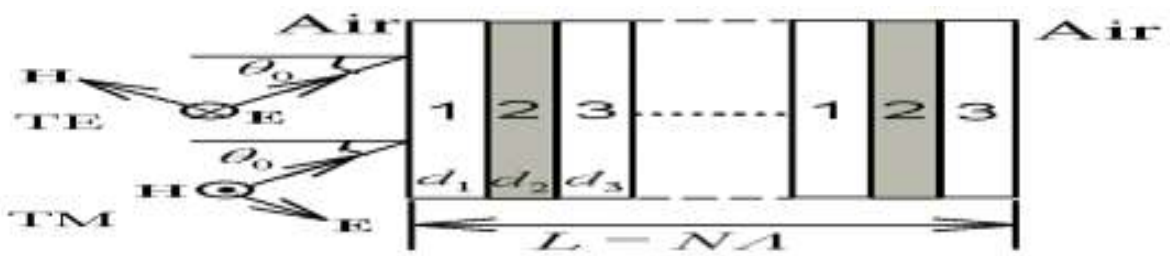

Figure 1: Schematic diagram of the ternary metallic photonic crystals

The permittivity of the metal in Drude model can be written as

$\varepsilon_{2}(\omega)=1-\omega_{\mathrm{p}}^{2} / \omega^{2}+\mathrm{j} \gamma \omega$

where $\omega_{\mathrm{p}}$ is the plasma frequency and $\Upsilon$ is the damping frequency. The index of refraction of a metal is given as $\mathrm{n}_{2}=\sqrt{ } \varepsilon_{2}$

For the TM wave, the characteristic matrix $M(\Lambda)$ is expressed as

$\mathrm{M}(\Lambda)==\left[\begin{array}{ll}M_{1 \mathbf{1}} & M_{1 \mathrm{z}} \\ M_{21} & M_{2 \mathrm{z}}\end{array}\right]_{=}\left[\begin{array}{ll}M_{1 \mathbf{1}} & M_{1 \mathrm{z}} \\ M_{21} & M_{2 \mathrm{z}}\end{array}\right] \prod_{l=\mathbf{1}}^{\mathrm{a}}\left[\begin{array}{cc}\cos \beta_{l} & \frac{1}{j p l} \sin \beta_{1} \\ -j p l \sin \beta_{1} & \cos \beta_{1}\end{array}\right]$

The matrix elements can be further calculated as follows ,

$\mathrm{M}_{11}=\cos \beta_{1} \cos \beta_{2} \cos \beta_{3}-\left(\mathrm{p}_{1} / \mathrm{p}_{2}\right) \sin \beta_{1} \sin \beta_{2} \sin \beta_{3}-\left(\mathrm{p}_{3} / \mathrm{p}_{2}\right) \cos \beta_{1} \sin \beta_{2} \sin \beta_{3}-\left(\mathrm{p}_{3} / \mathrm{p}_{2}\right) \sin \beta_{2} \cos \beta_{2} \sin \beta_{3}$

$\mathrm{M}_{12}=1 / \mathrm{jp} \mathrm{p}_{1} \sin \beta_{1} \cos \beta_{2} \cos \beta_{3}+1 / \mathrm{j} \mathrm{p}_{2} \cos \beta_{1} \sin \beta_{2} \cos \beta_{3}+1 / \mathrm{j} \mathrm{p}_{3} \cos \beta_{1} \cos \beta_{2} \sin \beta_{3}-\mathrm{p}_{2} / \mathrm{j} \mathrm{p}_{1} \mathrm{p}_{3} \sin \beta_{1} \sin \beta_{2} \sin \beta_{3}$

$M_{21}=-j p_{1} \sin \beta_{1} \cos \beta_{2} \cos \beta_{3}-\mathrm{j} p_{2} \cos \beta_{1} \sin \beta_{2} \cos \beta_{3}-\mathrm{j} \mathrm{p}_{3} \cos \beta_{1} \cos \beta_{2} \sin \beta_{3}+\mathrm{p}_{1} \mathrm{p}_{3} / \mathrm{p}_{2} \sin \beta_{1} \sin \beta_{2} \sin \beta_{3}$

$\mathrm{M}_{22}=\cos \beta_{1} \cos \beta_{2} \cos \beta_{3}-\mathrm{p}_{1} / \mathrm{p}_{2} \sin \beta_{1} \sin \beta_{2} \cos \beta_{3}-\mathrm{p}_{2} / \mathrm{p}_{3} \cos \beta_{1} \sin \beta_{2} \sin \beta_{3}-\mathrm{p}_{1} / \mathrm{p}_{2} \sin \beta_{1} \cos \beta_{2} \sin \beta_{3}$

Here $\beta_{1}=\mathrm{k}_{0} \mathrm{n}_{1} \mathrm{~d}_{1} \cos \Theta_{1}$ and $\mathrm{p}_{\mathrm{l}}=\mathrm{n}_{1} \cos \Theta_{\mathrm{l}}$. where $\mathrm{l}=1,2$, and 3, respectively, $\mathrm{k}_{0}=\omega \sqrt{\mu_{0} \varepsilon_{0}}$ is the free-space wave number. The reflectance can be written as $\mathrm{R}=\mathrm{r} * \mathrm{r}$,

Therefore the reflection coefficient $\mathrm{r}$ of the system is given by

$$
\frac{\left(m_{1_{1}}+m_{1 \mathrm{z}} p_{0}\right) p_{0}-\left(m_{21}+m_{2 \mathrm{z}} p_{0}\right)}{\left(m_{1_{1}}+m_{1 \mathrm{z}} p_{0}\right) p_{0}+\left(m_{2_{1}}+m_{2 \mathrm{z}} p_{0}\right)}
$$

where $\mathrm{p}_{0}=\mathrm{n}_{0} \mathrm{cos}$. The above formulations can also be applied for the $\mathrm{TM}$ wave by a simple replacement of $\mathrm{pl}=\cos / \mathrm{nl}$ where $\mathrm{l}=0,1,2$ and 3 respectively.

\section{RESULT AND DISCUSSION}

In this present communication, we have consider the ternary periodic structure (air\Ag lair) having layers 1 and 3 with the air materials whose refracting indices and thicknesses are $n_{1}=1.0, d_{1}=90 m m$ and $n_{3}=1.0$, $d_{3}=90 \mathrm{~mm}$, respectively. The metallic layer has been taken as silver (Ag) with the plasma frequency $\omega_{\mathrm{p}}=2$ $\pi \times 2.175 \times 10^{15} \mathrm{rad} / \mathrm{s}$ and the damping frequency is $\gamma=2 \pi \times 4.35 \times 10^{12} \mathrm{rad} / \mathrm{s}$ [7] which shown in figure 2 . The figure shows $\mathrm{R}$ (solid line) / $\mathrm{T}$ (dotted line) versus wavelength, where $\mathrm{R}$ and $\mathrm{T}$ means reflectance and transmission respectively. The wave length ranges are found from $4000 \mathrm{~A}^{0}$ to $1200 \mathrm{~A}^{0}$ for $\mathrm{TE}$ mode and also from $4000 \mathrm{~A}^{0}$ to $1200 \mathrm{~A}^{0}$ for TM mode. The $\mathrm{R}(\mathrm{T})$ for the considered structure is same for TE mode and TM mode. The completed reflectance or zero transmittance range is obtained from $7800 \mathrm{~A}^{0}$. The reflectance and the transmitted show the band gap near about $4200 \mathrm{~A}^{0}$ for the wavelength $4000 \mathrm{~A}^{0}$ $12000 \mathrm{~A}^{0}$ which is in visible region. Now for same structure, we have increased the thickness of $\mathrm{Ag}$ which is about $20 \mathrm{~nm}$ with other parameters are same which is shown in figure 3 . 


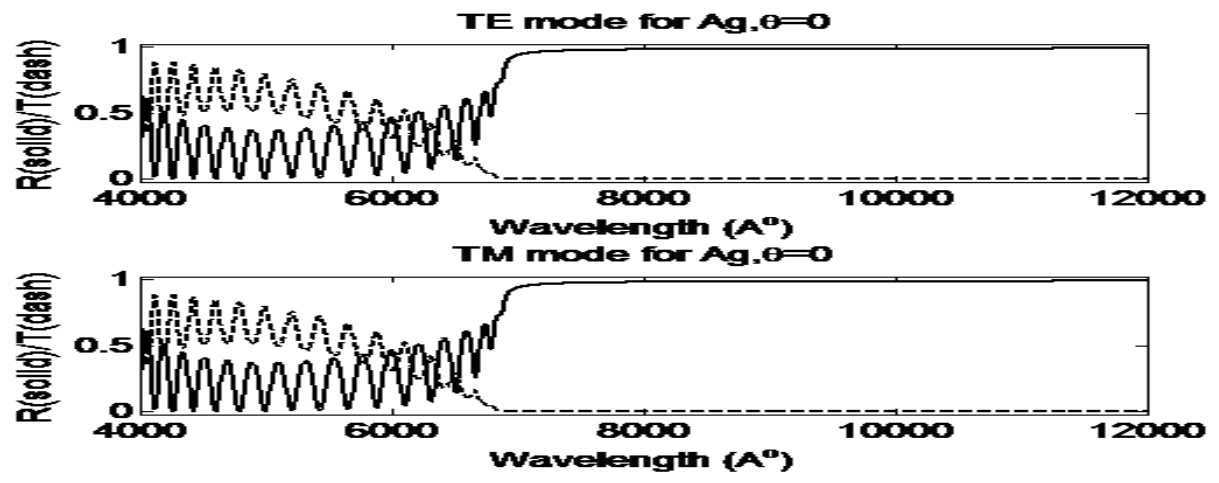

Figure 2: Reflectance/Transmitted versus wavelength of considered structure for (a) TE mode \& (b) TM mode.

Figure 3: shows the reflectance and transmittance for the considered structure for TE and TM modes at normal incidence which are the same. When the thickness of the Ag metal is increased, the band gap or reflectance is increased for very large range of wavelength. The band gap is found about
$600 \mathrm{~nm}$ for the air\Aglair ternary periodic structure with thickness $20 \mathrm{~nm}$. Thus we concluded that the thickness of the metal is increased, the reflection or forbidden band gap is enhanced due to the properties of the metal.
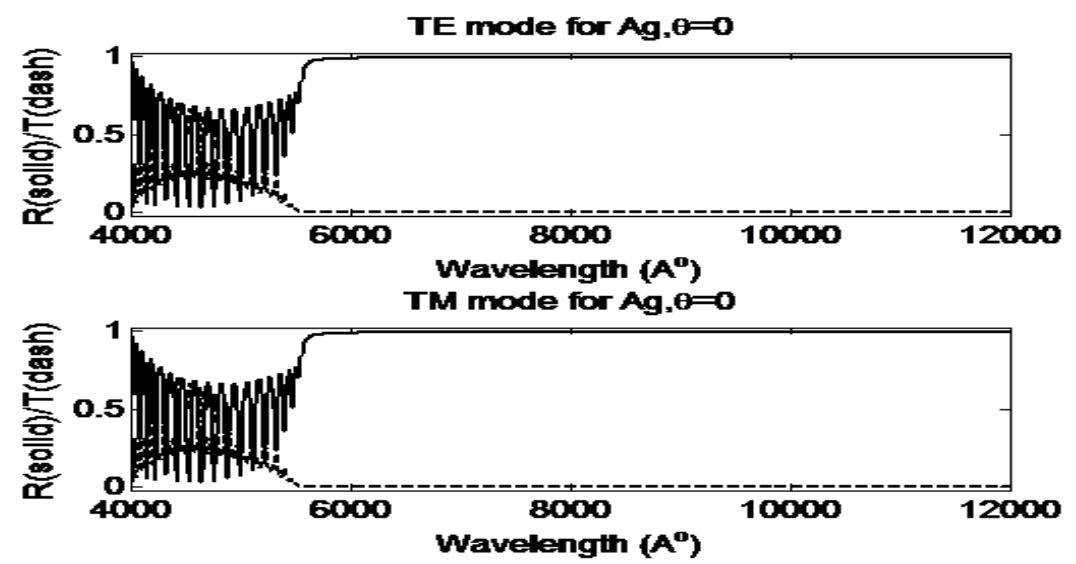

Figure.3: Reflectance/Transmitted versus wavelength of considered structure for (a) TE mode \& (b) TM mode

Again we have increased the thickness of the Ag for the same structure; we observed that the reflectance is enlarged as compare to the thickness of Ag 50nm. In figures 2, 3 and 4, we have taken $\mathrm{Ag}$ as a ternary structure with thicknesses $10 \mathrm{~nm}, 20 \mathrm{~nm}$ and $50 \mathrm{~nm}$ respectively which shows that the reflectance is increased if the thickness of the $\mathrm{Ag}$ metal is increased. For the same structure of the thickness of Ag metal is increased to $90 \mathrm{~nm}$, the reflectance is found $100 \%$ for all the range of the wavelength as shown in the figure 5 . Thus we have concluded from the above calculations that when the thickness of the Ag metal in ternary structure is increased then the reflectance is also increased and transmission for the large thickness is negligible. It means the large transmission is possible when the thickness of the Ag metal in ternary periodic structure should be small i.e. $10 \mathrm{~nm}$ or $20 \mathrm{~nm}$.

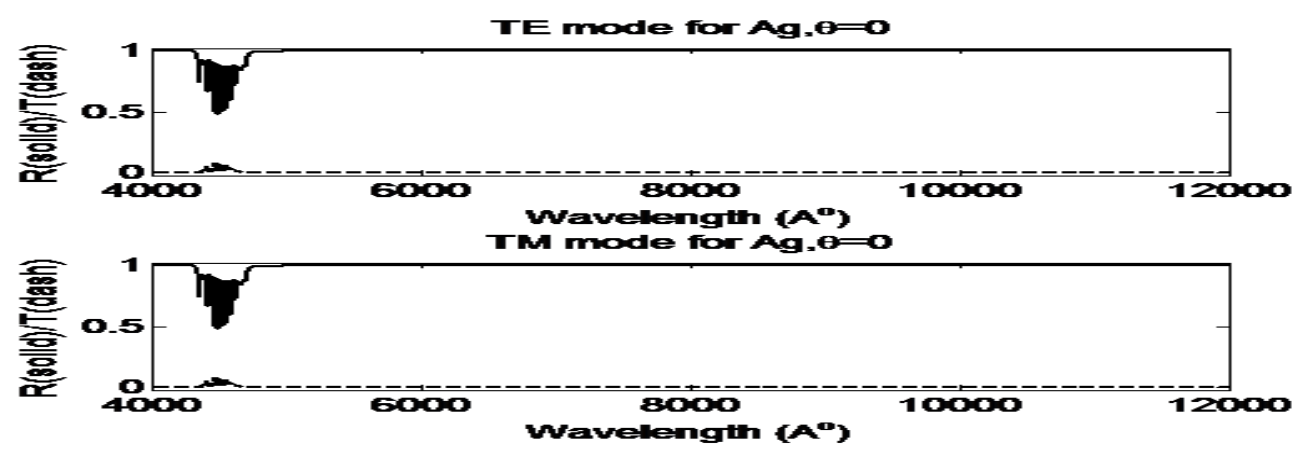

Figure 4: Reflectance/Transmitted versus wavelength of considered structure for (a) TE mode \& (b) TM mode 


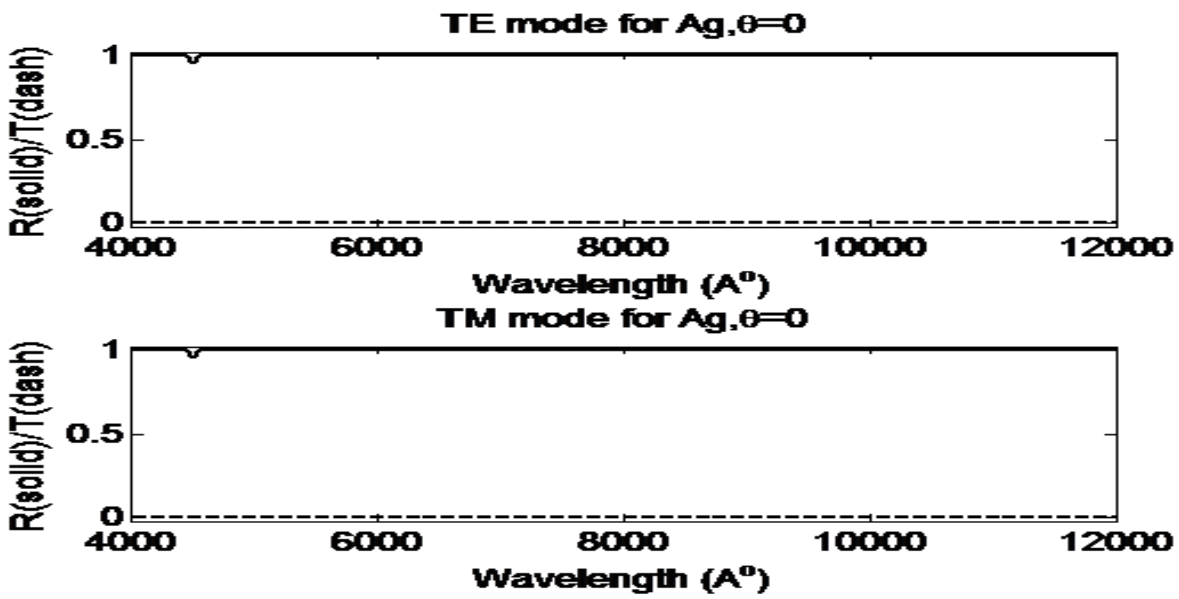

Figure 5: Reflectance/Transmitted versus wavelength of considered structure for (a) TE mode \& (b) TM mode

\section{ACKNOWLEDGEMENT:}

Dr G N Pandey is thankful to Dr. Khem B Thapa, Department of Physics, B.B.A.University, (A Central University) Lucknow, Uttar Pradesh, for his interest in research and constant encouragement.

\section{REFERENCES}

[1] Yablonovitch, E., "Inhibited spontaneous emission in solid state physics and electronics," Phys. Rev. Lett., Vol. 58, 20592062, 1987.

[2] John, S., "Strong localization of photons in certain disordered lattices," Phys. Rev. Lett., Vol. 58, 2486-2489, 1987.

[3] J. W. S. Rayleigh; "On the remarkable phenomenon of crystalline reflexion described by Prof. Stokes".Phil. Mag.26, 256 (1888).

[4] Born, M. and E. Wolf, "Principles of Optics", Cambridge, London, 1999.

[5] S.J. Orfanidis, "Electromagnetic Waves and Antennas", R. University, (2008).

[6] P. Yeh "Optical wave in layered media", J. Wiley and Sons, Singapore (1991).

[7] C. J. Wu, Y. H. Chung, B. J. Syu and T. J. Yang, "Band Gap Extension in a OneDimensional Ternary Metal-Dielectric Photonic Crystal" Progress in Electromagnetic Research, 102, 81, (2010).

[8] P. Drude, Theory of Optics (Longmans, Green, New York, 1922; Dover, New York, (1968).

[9] G.N. Pandey, Khem. B. Thapa, and S .P. Ojha, "Reflection Properties of One Dimensional Magnetic Photonic Crystals", Journal of Ovonic Research (U S A), Vol.6, 87-92, (2010).

[10] G.N. Pandey, K. B. Thapa and S.P. Ojha, "Ominidirectional Reflection Band in One Dimensional Plasma Photonic Crystal",
Optik - International Journal for Light and Electron Optics, Volume 124, Issue 18, Pages.3396-340 (2013)

[11] G. N. Pandey and S. P. Ojha, " Band Structure, Group velocity, Effective group index and Effective phase index of one Dimensional Plasma Photonic Crystal", Optik - International Journal for Light and Electron Optics, Volume 124, Issue 18, Pages 3514-3519,(2013).

[12] G. N. Pandey,J. P. Pandey, A, K, Mishra and S. P. Ojha, "Three Dimensional Reflectance Properties of Plasma Dielectric Photonic Crystal" Proceedings of the International Conference on "Condensed Matter \& Applied Physics" (ICC- 2015)" AIP Conference Proceedings, Volume 1728, pp 020312-1-020312-4, (2016).

[13] G. N. Pandey, Narendra Kumar, Khem B Thapaand S. P. Ojha, "Reflectance Properties of One-Dimensional MetalDielectric Ternary Photonic Crystal" Proceedings of the International Conference on "Condensed Matter \& Applied Physics" (ICC- 2015)" AIP Conference Proceedings Volume 1728, 020310-1-020310-3, (2016).

[14] G. N. Pandey, J. P. Pandey, U. K. Pandey, BhagyashreeSancheti and S. P. Ojha, "Three Dimensional Reflectance Properties of Superconductor- Dielectric Photonic Crystal" Proceedings of the International Conference on "Condensed Matter \& Applied Physics" (ICC- 2015)” AIP Conference Proceedings, 1728, pp 0203061-020306-4, (2016).

[15] Khem B. Thapa, S.K. Singh, S.K. Srivastava, Om Prakash, G. N. Pandey, U.N. Singh and S.P. Ojha, "Reflection properties, anomalous group velocity and negative refraction index in one-dimensional Plasma Photonic Crystal" Proceedings of "Seventh 
International Conference on

Optoelectronics, Fiber Optics and Photonics (PHOTONICS 2004)", 9-11 December, pp. -OMD -P-360 (2004)

[16] S. K. Srivastava, G. N. Pandey, Khem B. Thapa, S. K. Awasthi and S.P. Ojha, “ Design of Omnidirectional Optical Reflector using Plasma-Dielectric Photonic Crystal" Proceedings of "National workshop on Optoelectronic Material and Devices (AOMD-2007)", 27-29 Dec, pp. - 305-311, (2007)

\footnotetext{
International Journal of Engineering Research and Applications (IJERA) is UGC approved Journal with S1. No. 4525, Journal no. 47088. Indexed in Cross Ref, Index Copernicus (ICV 80.82), NASA, Ads, Researcher Id Thomson Reuters, DOAJ.

G. N. Pandey. "Photonic Band Gap in One-Dimensional Ternary Metal-Dielectric Photonic Crystal." International Journal of Engineering Research and Applications (IJERA) 7.7 (2017): 87-91.
} 